C-A/AP/\#230

March 2006

\title{
Simple Estimates of Collisional Excitation Cross Sections
}

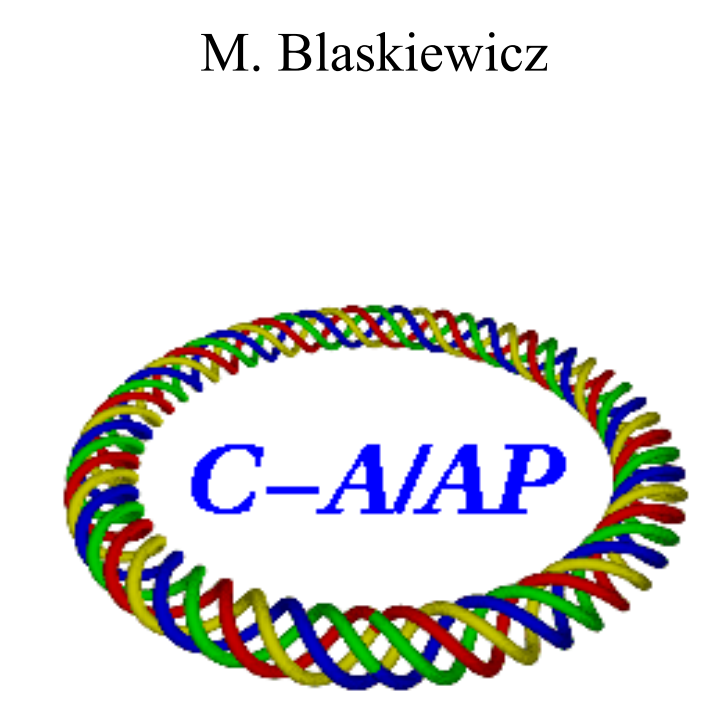

Collider-Accelerator Department Brookhaven National Laboratory

Upton, NY 11973 


\title{
Simple estimates of collisional excitation cross sections
}

\author{
M. Blaskiewicz* \\ $B N L$, Upton NY 11973, USA
}

\begin{abstract}
Transverse emittance reduction has been observed in the AGS with helium like gold [1]. Additionally, the extracted emittance is always close to $10 \pi \mathrm{mm}$ - mrad regardless of the care with which the beam is accelerated [2]. It has been suggested [3, 4] that the cooling is due to collisionally excitating the electronic levels with the subsequently emitted photon removing energy from the system. Simple estimates for the collisional excitation of the electronic levels and the cooling rates based upon them are presented. I find no evidence supporting the hypothesis.
\end{abstract}

\section{INTRODUCTION}

Typical parameters for an $\mathrm{Au}^{+77}$ bunch on the AGS injection porch are given in Table I. Since the bunch is nonrelativistic the rms transverse velocity is $\sigma\left(v_{x}\right)=\omega_{0} Q_{x} \sigma(x)=4 \times 10^{4} \mathrm{~m} / \mathrm{s}$, and the rms longitudinal velocity is $\sigma\left(v_{s}\right)=C f_{s} \omega_{0} \sigma(t)=2.7 \times 10^{5} \mathrm{~m} / \mathrm{s}$. With these $\mathrm{rms}$ velocities the typical kinetic energy in the comoving frame is $\delta E=M\left[\sigma\left(v_{s}\right)^{2} / 2+\sigma\left(v_{x}\right)^{2}\right]=M c^{2} \sigma(v / c)^{2} / 2 \approx 80 \mathrm{keV}$. Cooling the beam requires a mechanism to reduce $\delta E$. Inelastic collisons between the ions in the bunch are a possible candidate for this energy loss and we go on to consider this possibility.

\section{ASSUMPTIONS AND KINEMATICS}

We will treat the motion of the nuclei classically and the electrons and photons quantum mechanically. Toward this end consider Figure 1. In Fig $1 \mathrm{~A}$, which is the center of mass frame, each ion has a velocity of magnitude $v_{0}$ and the total energy is $E_{i}=M v_{0}^{2}$. During the collision one of the ions is excited and radiates a photon of energy $\Delta E$, and momentum $\Delta E / c$. In the final state let the ion velocities be $\mathbf{v}_{1}$ and $\mathbf{v}_{2}$ and suppose that the photon travels in direction $\mathbf{n}$. Conservation of momentum and energy read

$$
M\left(\mathbf{v}_{1}+\mathbf{v}_{2}\right)+\mathbf{n} \Delta E / c=0
$$

and

$$
M\left(v_{1}^{2}+v_{2}^{2}\right) / 2+\Delta E=M v_{0}^{2} .
$$

TABLE I: AGS injection parameters for $\mathrm{Au}^{+77}$.

\begin{tabular}{lc}
\hline \hline parameter & value \\
\hline circumference & $C=807$ \\
Lorentz factor & $\gamma=1.10$ \\
revolution period & $T_{0}=6.4 \mu \mathrm{s}$ \\
angular revolution frequency & $\omega=2 \pi / T_{0}=9.8 \times 10^{5} \mathrm{~s}^{-1}$ \\
nominal betatron tunes & $Q_{x}=Q_{y}=8.2$ \\
rms beam radius & $\sigma(x)=\sigma(y)=5 \mathrm{~mm}$ \\
$h=24$ RF voltage & $V_{R F}=280 \mathrm{kV}$ \\
synchrotron frequency & $f_{s}=6.8 \mathrm{kHz}$ \\
rms bunch length & $\sigma(t)=50 \mathrm{~ns}$ \\
ions per bunch & $N_{b}=5 \times 10^{8}$ \\
nuclear mass and charge & $M=185 \mathrm{GeV} / \mathrm{c}^{2}, \mathrm{Z}=79$ \\
\hline \hline
\end{tabular}

*Electronic address: blaskiewicz@bnl.gov 

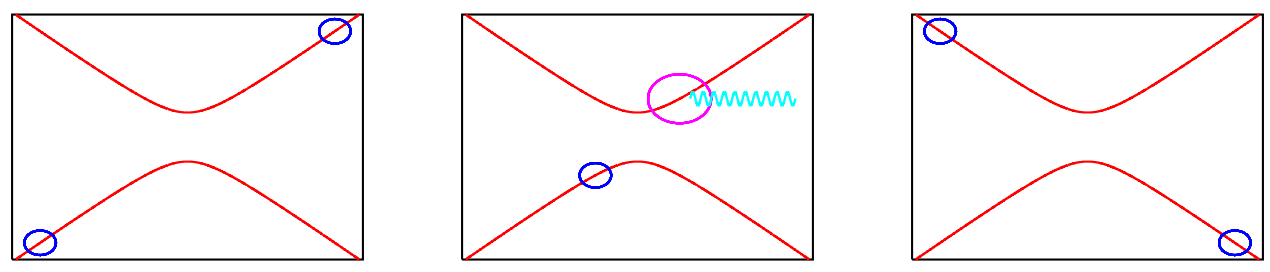

FIG. 1: A schematic of collisional excitation. Initially (A, left) the two ions are in the electronic ground state. As they approach (B, center) one of the ions undergoes an electronic excitation and radiates a photon of energy $\Delta E$. Finally (C, right) the ions separate with the total kinetic energy reduced by $\Delta E$.

We now show that the photon momentum in $\mathrm{Eq}(1)$ can be neglected for $v_{0} \ll c$. From Eq (1) the maximum difference in the final speeds of the particles occurs when $\hat{n}$ is parallel to either $\mathbf{v}_{1}$ or $\mathbf{v}_{2}$. For definiteness take $\hat{n}$ parallel to $\mathbf{v}_{2}$. Then the final speeds are related by $v_{2}=v_{1}-\Delta E / M c$. Substituting this in equation (2) one finds that the maximum speed for a particle in the final state is

$$
v_{\text {max }}=\sqrt{v_{0}^{2}-\frac{\Delta E}{M}\left[1-\frac{\Delta E}{M c^{2}}\right]+\left[\frac{\Delta E}{2 M c}\right]^{2}}+\frac{\Delta E}{2 M c} .
$$

Additionally, from Eq (1) we have $\left|v_{1}-v_{2}\right| \leq\left|\mathbf{v}_{1}+\mathbf{v}_{2}\right|=\Delta E / M c$ when all photon directions are considered. Therefore

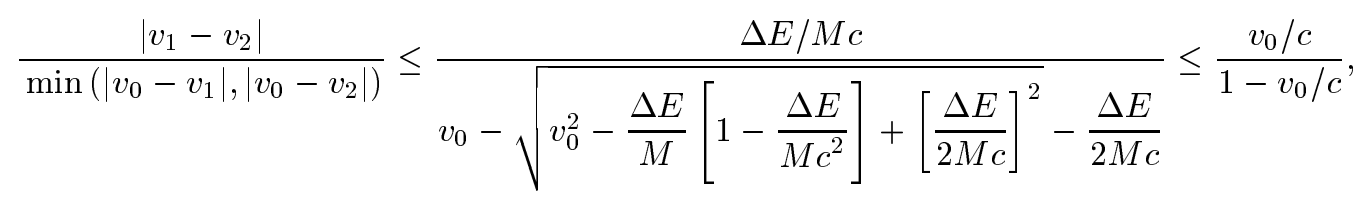

where the final inequality follows from multiplying top and bottom by $v_{0}+v_{\max }-\Delta E / M c$ and simplifying.

For the problem at hand $v_{0} / c<<1$ so that we may take $v_{1}=v_{2}$ and the change in velocity of either ion is $v_{0}-\sqrt{v_{0}^{2}-\Delta E / M}$. From a physical standpoint one may suppose that the ion velocities change when the ion is excited and that the subsequent photon emission doesn't change the velocity significantly.

While the final state velocites can be calculated, the details of the excitation process need to be treated quantum mechanically so that a classical description of the ion trajectories cannot be obtained. A full blown quantum mechanical description will not be attempted here. Instead, the excitation probabilities will be obtained by assuming that the ions undergo classical Rutherford scattering. This yields a time dependent classical potential which can lead to excitation of the electrons.

To obtain the ion trajectories let $v_{r}=2 v_{0}$ be the relative velocity of the ions before the collision and let $b$ be the impact parameter. The distance of closest approach between the nuclei satisfies (cgs units,[5] p108)

$$
\frac{1}{r_{\min }}=\frac{2 Z^{2} e^{2}}{M v_{r}^{2} b^{2}}\left[\sqrt{1+\left(\frac{M v_{r}^{2} b}{2 Z^{2} e^{2}}\right)^{2}}-1\right] .
$$

The radial acceleration is a maximum at $r_{\min }$ and is given by

$$
\ddot{r}_{\text {max }}=\frac{2 Z^{2} e^{2}}{M r_{\text {min }}^{2}} \sqrt{1+\left(\frac{M v_{r}^{2} b}{2 Z^{2} e^{2}}\right)^{2}} .
$$

When $r=r_{\min }$ the direction of the internuclear axis is rotating at its maximum rate and is given by

$$
\dot{\theta}_{\max }=\frac{b v_{r}}{r_{\min }^{2}}
$$

For purposes of easy calculation note that

$$
\frac{M v_{r}^{2} b}{Z^{2} e^{2}}=\left(\frac{v_{r}}{c}\right)^{2} \frac{b}{r_{c}}
$$


where $r_{c}$ is the classical particle radius, $r_{c}=\left(Z^{2} / A\right) 1.54 \times 10^{-18}$ meters which yields $r_{c}=4.63 \times 10^{-17} \mathrm{~m}$ for helium-like gold.

\section{ELECTRONIC DIPOLE TRANSITIONS}

Consider the orders of magnitude in the problem. During the collision the electric field at one nucleus due to the other is $\mathbf{E}(t)=\hat{n} Z e / r^{2}(t)$, where $\hat{n}$ is a unit vector. For $v_{r}=10^{6} \mathrm{~m} / \mathrm{s}$ and $b=0, r_{\text {min }}=0.17 \times 10^{-10} \mathrm{~m}$. The electron cloud of helium-like gold has an extent $\sim a_{0} / Z$ where $a_{0}=0.53 \times 10^{-10} \mathrm{~m}$ is the Bohr radius. The ratio of the extent of the electron cloud to the distance of closest approach is $\lesssim 0.04$. Therefore, the electron clouds of the two ions remain well separated and we consider the evolution of each ion independently. We take our coordinate origin as one of the nuclei and neglect the effects of rotation and acceleration of the nucleus. Therefore the interaction hamiltonian is $H_{i}(t)=z e^{2} Z / R^{2}(t)$ where $z$ is the electron coordinate along a fixed axis and we have gone over to $R$ to describe the internuclear distance. The screening effects are small so consider a single electron with hamiltonian $H=H_{0}+H_{i}$ Work in the two state approximation with $H_{0}\left|0>=E_{0}\right| 0>, H_{0}\left|1>=E_{1}\right| 1>$, and interaction energy $T=\left\langle 0\left|H_{i}\right| 1\right\rangle$. Choose a basis so that $T$ is real and $E_{0}<E_{1}$. Next, for a fixed value of $R$, define the adiabatic states $\mid->$ and $\mid+>$.

$$
\begin{gathered}
|->=\cos \chi| 0>-\sin \chi \mid 1> \\
|+>=\sin \chi| 0>+\cos \chi \mid 1>
\end{gathered}
$$

where

$$
\begin{array}{r}
<0\left|H_{0}+H_{i}\right|->=E_{-}<0 \mid->=E_{0} \cos \chi-T \sin \chi=E_{-} \cos \chi \\
<1\left|H_{0}+H_{i}\right|->=E_{-}<1 \mid->=T \cos \chi-E_{1} \sin \chi=-E_{-} \sin \chi
\end{array}
$$

and a similar equations hold for $\mid+>$. Solving the algebra yields

$$
E_{ \pm}=\left(E_{0}+E_{1}\right) / 2 \pm \sqrt{\left(E_{0}-E_{1}\right)^{2} / 4+T^{2}}
$$

with

$$
\tan (2 \chi)=2 T /\left(E_{1}-E_{0}\right)
$$

Define the phases

$$
\Phi_{ \pm}=\int_{0}^{t} E_{ \pm}\left(t_{1}\right) d t_{1} / \hbar
$$

and let the wavefunction drscribing the system be

$$
\left|\Psi>=C_{-}(t)\right|->\exp \left[i \Phi_{-}(t)\right]+C_{+}(t) \mid+>\exp \left[i \Phi_{+}(t)\right]
$$

with $C_{-}(-\infty)=1$. At time $t,\left|C_{+}(t)\right|^{2}$ is the probability that the electron is in the excited state. Remember that both $\mid+>$ and $\mid->$ depend on time, since $\chi$ does. The equations for $C_{+}$and $C_{-}$follow from

$$
<+\left|i \hbar \frac{\partial}{\partial t}-H_{0}-H_{i}\right| \Psi>=0
$$

and

$$
<-\left|i \hbar \frac{\partial}{\partial t}-H_{0}-H_{i}\right| \Psi>=0 .
$$

After taking the inner products one finds

$$
\begin{aligned}
& \dot{C}_{+}=-\dot{\chi} C_{-} \exp \left(i\left[\Phi_{+}-\Phi_{-}\right]\right) \\
& \dot{C}_{-}=+\dot{\chi} C_{+} \exp \left(i\left[\Phi_{-}-\Phi_{+}\right]\right) .
\end{aligned}
$$

By construction $E_{+}>E_{-}$so for times well before the collision we may take $C_{-}=1$ and $C_{+}=0$. Under the supposition that $C_{+}$remains small compared to 1 a first approximation is given by

$$
C_{+}(t)=-\int_{-\infty}^{t} d t_{1} \dot{\chi}\left(t_{1}\right) \exp \left(i\left[\Phi_{+}\left(t_{1}\right)-\Phi_{-}\left(t_{1}\right)\right]\right) .
$$




\begin{tabular}{ccc}
\hline \hline$m_{e} c^{2}-E_{n, j} \mathrm{keV}$ & $n$ & $j$ \\
\hline 93.46 & 1 & $1 / 2$ \\
23.93 & 2 & $1 / 2$ \\
21.69 & 2 & $3 / 2$ \\
\hline \hline
\end{tabular}

TABLE II: Binding energies for $Z=79$. Both the $2 S_{1 / 2}$ and $2 P_{1 / 2}$ states have $n=2, j=1 / 2$. The energy degeneracy is removed by the Lamb shift.

Using non-relativistic wavefunctions the interaction energy is given by

$$
T=<1\left|H_{i}\right| 0>=G a_{0} e E(t) / Z
$$

where $G$ is a pure number depending on which orbitals are involved. For $1 S \rightarrow 2 P, G=8(2 / 3)^{5} / \sqrt{2} \approx 0.745$; for $2 S \rightarrow 2 P, G=3$.

A photon can be emitted either during the collision or well after the collision. Consider the second case and also assume $\chi \ll 1$ during the collision so that $\left(E_{+}-E_{-}\right) \approx E_{1}-E_{0} \equiv \hbar \omega$ and $\tan (2 \chi) \approx 2 \chi$. Then

$$
\begin{aligned}
C_{+}(\infty) & \approx-\int_{-\infty}^{\infty} \frac{<1\left|H_{i}\right| 0>}{\hbar} \exp (i \omega t) d t \\
& =\frac{G e a_{0}}{\hbar Z} \int_{-\infty}^{\infty} E(t) \exp (i \omega t) d t \\
& \approx \frac{G e a_{0}}{\hbar Z} \int_{-\infty}^{\infty} \frac{Z e}{\left[r_{\min }+a_{\max } t^{2} / 2\right]^{2}} \exp (i \omega t) d t \\
& \approx \frac{G e^{2} a_{0}}{\hbar r_{\min }^{2}} \int_{-\infty}^{\infty} \frac{1}{1+a_{\max } t^{2} / r_{\min }} \exp (i \omega t) d t \\
& \approx \pi \frac{G e^{2} a_{0}}{\hbar r_{\min }^{2}} \tau_{c} \exp \left(-\omega \tau_{c}\right),
\end{aligned}
$$

where $\tau_{c}=\sqrt{r_{\min } / a_{\max }}$ is the characteristic time scale of the collision. For a given $v_{r}$ the characteristic time is minimum for $b=0, \tau_{c, \text { min }}=\sqrt{32}\left(c / v_{r}\right)^{3} r_{c} / c$.

For a hydrogen-like atom with nuclear charge $Z e$ the energies are [7] p75.

$$
E_{n, j}=m_{e} c^{2}\left(1+\left[\frac{Z \alpha}{n-j-1 / 2+\sqrt{(j+1 / 2)^{2}-Z^{2} \alpha^{2}}}\right]^{2}\right)^{-1 / 2}
$$

where $\alpha=e^{2} / \hbar c=1 / 137.036$ is the fine structure constant, $n=1,2, \ldots$ is the principle quantum number and $j=1 / 2,3 / 2, \ldots n-1 / 2$ is to total angular momentum of the state. Table II gives a few values for $Z=79$. For helium-like gold the $1 S \rightarrow 2 P$ transition has excitation energy $71.8 \mathrm{keV}$ while the $2 S \rightarrow 2 P$ transition has excitation energy $2.24 \mathrm{keV}$. Taking $v_{r}=1 \times 10^{6} \mathrm{~m} / \mathrm{s}$ we find $\omega \tau_{c, \min }=75$ for the $2 S \rightarrow 2 P$ transition. So in this approximation there is essentially no probability of emitting a photon with either helium-like or lithium-like gold ions in the AGS.

Radiative corrections remove the degeneracy between the the $2 S_{1 / 2}$ and $2 P_{1 / 2}$ states, which both have have $n=2$, $j=1 / 2$. For lithium-like uranium the measured value is $E_{2 S_{1 / 2}}-E_{2 P_{1 / 2}}=280.6 \mathrm{eV}$ [8]. The energy shift is given by $[7][\mathrm{p} 365]$

$$
E_{2 S_{1 / 2}}-E_{2 P_{1 / 2}}=\frac{m_{e} c^{2} \alpha^{4} Z^{5}}{6 \pi}\left(\ln \left[\frac{<E_{2 P}>}{Z^{2} \alpha^{2}<E_{2 S}>}\right]+\frac{91}{120}\right)
$$

where $\left\langle E_{2 S}>\right.$ and $\left\langle E_{2 P}>\right.$ are defined in terms of expectation values for energy shifts due to interactions with the zero-point fluctuations [7][p 364]. We assume that lithium-like gold is sufficiently close to lithium-like uranium so that 


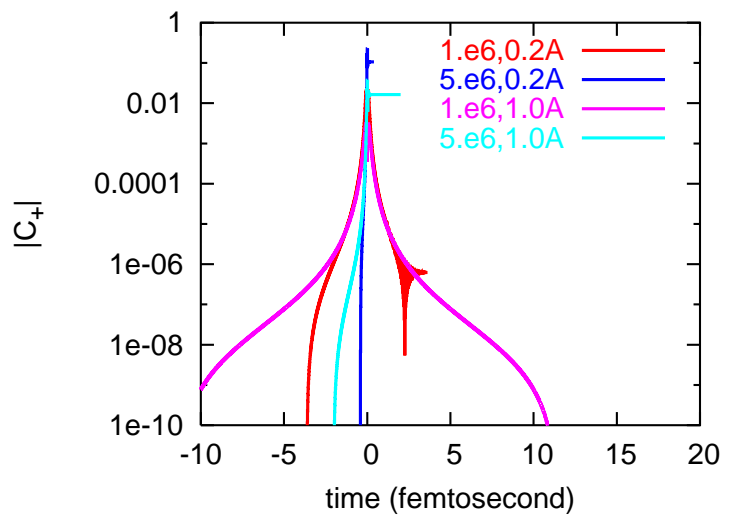

FIG. 2: Numerically obtained plots of $\left|C_{+}\right|$for the Lamb transition. Various values of $v_{r}(\mathrm{~m} / \mathrm{s})$ and $b$ (anstroms) are shown.

only the leading $Z^{4}$ scaling is important. This gives $E_{2 S_{1 / 2}}-E_{2 P_{1 / 2}} \approx-149 \mathrm{eV}$. Taking the same relative velocity as before we get $\omega \tau_{c} \approx 5$. However, for these same parameters $T /\left(E_{1}-E_{0}\right) \approx 5.8$ at closest approach so equation (20) is inapplicable. Numerical integration of (15) will be used for this case. Representative results are shown in Figure 2.

Once $C_{+}\left(\infty, b, v_{r}\right)$ is obtained the total excitation cross section is given by

$$
\sigma\left(v_{r}\right)=2 \pi \int_{0}^{\infty} b d b\left|C_{+}\left(\infty, b, v_{r}\right)\right|^{2}
$$

For the three transitions considered the results are very small. For $v_{r}=10^{6} \mathrm{~m} / \mathrm{s}$ the $2 S_{1 / 2} \rightarrow 2 P_{1 / 2}$ cross section is $4 \times 10^{-33} \mathrm{~m}^{2}$ and this rises to $5 \times 10^{-26} \mathrm{~m}^{2}$ for $v_{r}=2 \times 10^{6} \mathrm{~m} / \mathrm{s}$. The cross sections for the other two transitions are even smaller.

It might be possibile that the photon is emitted during the collision. To estimate the probability of this we use

$$
P_{\gamma} \approx \max _{t}\left|C_{+}(t)\right|^{2} \tau_{c} / \tau_{r}
$$

where $\tau_{r}$ is the radiative lifetime of the excited state. As a rough approximation to $\tau_{r}$ we take the nonrelativistic quantum mechanical expression [9] p179,

$$
\frac{1}{\tau_{r}}=\frac{4 \alpha \omega^{3} D^{2}}{3 c^{2}}
$$

Where $D=G a_{0} / Z$ is the electric dipole moment of the transition for radiation of frequency $\omega$. We estimate $\left|C_{+}(t)\right| \sim$ $|\dot{\chi}| / \omega$ and obtain cross sections $\sim 10^{-30} \mathrm{~m}^{2}$ for the relevant parameter ranges.

\section{CONCLUSIONS}

The possibility of electronic excitation of gold ions in the AGS due to collisions has been considered. We found cross sections $\sigma \lesssim 10^{-30} \mathrm{~m}^{2}$ for reasonable parameters. For typical particle densities of $n=10^{11} \mathrm{~m}^{-3}$ and a relative velocity of $v_{r} \lesssim 10^{6} \mathrm{~m} / \mathrm{s}$ the mean free time between collisions is $\tau_{m f}=1 /\left(n \sigma v_{r}\right) \gtrsim 10^{13} \mathrm{sec}$ which is 13 orders of magnitude too long to be of consequence.

\section{Acknowledgements}

Thanks to Thomas Roser and Dejan Trbojevic for useful discussions and encouragement.

[1] Wilem Van Asselt private communication circa 1995. 
[2] Leif Ahrens private communication.

[3] Thomas Roser private communication.

[4] K. Kilian, Proceedings of the Workshop on Electron Cooling and New Cooling Techniques, World Scientific, Singapore, New Jersey, London, Hong Kong, p 238, 1990.

[5] H. Goldstein, Classical Mechanics Addison-Wesley, 1981.

[6] See eg L.I. Schiff Quantum Mechanics McGraw-Hill, (1968).

[7] C. Itzykson and J.B. Zuber Quantum Field Theory McGraw-Hill, (1980).

[8] J. Schweppe, A. Belkacem, L.Blumenfeld, N. Claytor, B. Feinberg, H. Gould, V.E. Kostroun, L.Levy, S. Misawa, J.R. Mowatm H.H. Prior, PRL 66, 1434-1437, (1991).

[9] R. Louden, The Quantum Theory of Light Oxford, 1986. 